\title{
Introduction to the presidential symposium at the International Congress of Neuroethology, ICN 2018 in Brisbane, Australia
}

\author{
Catharine H. Rankin ${ }^{1}$
}

Received: 28 March 2020 / Revised: 1 April 2020 / Accepted: 3 April 2020 / Published online: 18 April 2020

c) Springer-Verlag GmbH Germany, part of Springer Nature 2020

The articles in this section of the present issue of Journal of Comparative Physiology- $A$ are from speakers at the 2nd Presidential Symposium of the Congress of the International Society for Neuroethology in Brisbane in June 2018. The speakers were invited to represent the diversity of research that can fall under the heading of Neuroethology. Speakers were chosen for the excellence of their research, for their ability to communicate the excitement and importance of the study of behavior, and to challenge the audience to think about behavior in new and different ways. Speakers were also requested to provide an article for JCP-A. A substantial portion of the travel costs for the Presidential Symposium speakers attending the Congress was offset by Springer-Verlag as well as by the International Society for Neuroethology and a generous personal contribution from Professor Friedrich and Ortrun Barth. We hope that these contributors (and others) will continue their critical support for the Presidential Symposium in future Congresses of the International Society of Neuroethology. The symposium serves as a kick-off for the meeting and sets the tone to top-quality presentations and exciting science.

Neuroethology strives to understand the neural basis of naturally occurring behavior. Understanding behavior is critical to understanding neuroscience in the context that behavior is the ultimate phenotype, the final read-out of the nervous system, and a primary target of evolution. For the uninitiated, behavior seems simple - however, modern techniques for studying behavior have shown us that the majority of behaviors have many, many degrees of freedom, and that a single behavior can serve multiple functions. In any given moment, behavior is sculpted by the nervous system, by the animals' experience, possibly the experience of the

Catharine H. Rankin

crankin@psych.ubc.ca

1 Department of Psychology and DM Centre for Brain Health, University of British Columbia, 2211 Wesbrook Mall,

Vancouver, BC V6T 2B5, Canada animals' parents and grandparents, and by the environment, and these are all impacted by the animals' evolutionary history. Although many researchers study behavior as part of their research programs, the hallmark of a neuroethologist is a focus on behavior and how a behavior is adaptive for the organism. In studies aimed at understanding the genetic and neural bases of behavior, the key is to keep going back to the behavior under study at every step along the way, confirming that the properties of the putative underlying mechanisms match the properties of the behavior. A 2017 position paper in Neuron by Krakauer et al. was titled "Neuroscience Needs Behavior: Correcting a Reductionist Bias", that was based on the premise that "Behavior is complex and nuanced. To really understand complexities of behavior one must see it as a meaningful endpoint and not a simple single binary unit."

To effectively study behavior, it begins with observing a simple behavior and thinking about how it can be operationally defined. Start the study using that operational definition; however, let the data refine your definition of the behavior as often as is necessary-as your understanding of the behavior grows, the definition may also evolve. Operationally defining behavior is a critical part of studying behavior. How you define the behavior will determine the results you find!

Too often these days in neuroscience, the holy grail is "mechanism", and behavior is seen as a simple observation that begins the quest for mechanism. Rarely are the mechanistic elements tested against the behavior to ensure that the features of the mechanisms (timing, intensity, frequency, etc.) match up with the parameters of the behavior. While perhaps easier to do poorly, studying behavior is at least as complicated and tricky to get right as physiology or molecular biology_-for example, tossing a mouse in a Morris Water Maze and measuring time to reach the platform are not as simple as it might outwardly seem. There are many cautionary tales of drowning mice, of effects of water temperature on performance, small environmental cues unnoticed by the experimenter that the mice can use. Good studies of behavior require a thorough 
understanding of the variables that can affect behavior and taking them into consideration in the research design.

The papers in this issue address a wide range of behavioral topics from insect to humans, from sensory abilities to sex differences. The speakers were all superb and the articles which they have written for this issue of JCP-A offer an intellectually stimulating array of ideas, new information, new hypotheses, and new ways to think about behavior and overall first-rate science. They are:

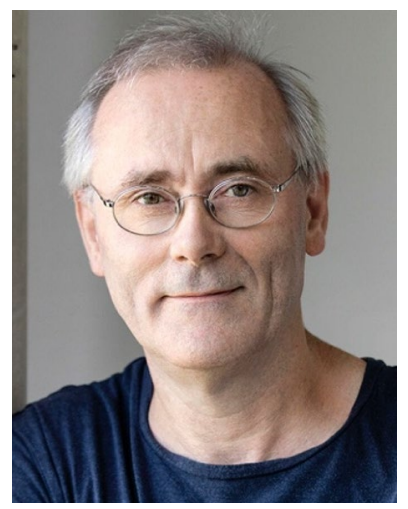

The symposium opened with a wonderful talk by Gary Lewin about a fascinating animal, the naked mole rat. If you do not know a lot about naked mole rats, then this review entitled "Independent evolution of pain insensitivity in African mole rats: origins and mechanisms" by Ewan St John Smith, Thomas J Park, and Gary Richard Lewin is a great place to start as it captures the current state of this most interesting field. Naked mole rats are one of only two groups of eusocial mammals! They live in large colonies underground and only a single queen reproduces. The large colonies living in burrows have a number of fascinating adaptations to this lifestyle. One of the more intriguing adaptations explored by Dr. Lewin is altered pain sensation. While most animals show a pain response if exposed to capsaicin or acidic solutions, these animals do not. In his review, Dr. Lewin offers explanations about how high population density in deep burrows leads to the evolution of diminished pain responses to some stimuli, but not others. Dr. Lewin presents evidence that adaptations in pain receptors are the result of mutations in several different molecules including the nerve growth factor TrK1A and the voltage gated sodium channel Nav1.7, as well as alterations in the wiring of the pain neurons and the absence of neuropeptides in critical sensory neurons.

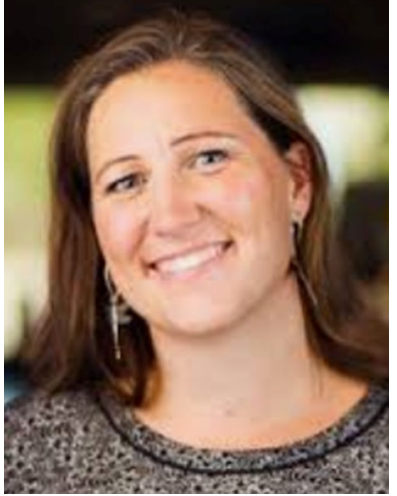

The second talk in the symposium described how dung beetles from different ecological environments navigate. This experimental report entitled "Straight-line orientation in the woodland-living beetle Sisyphus fasciculatus" by Lana Khaldy, Claudia Tocco, Marcus Byrne, Emily Baird, and Marie Dacke investigated mechanisms of orientation. Dung beetles forage for piles of animal dung. When they find such a pile, they carve off a ball and move it away from the main pile to avoid competitors. To date, the species of dung beetles studied have been from the savanna, and studies have shown that their primary orientation cue when they are moving their dung balls is the sun. In the study described in this paper, Dr. Dacke studied dung beetles from savanna-type environments as well as from woodland-type environments where the sun is rarely visible because of the density of the vegetation. These beetles used polarized light as their preferred orientation cue. Studying beetles both in the wild and in the lab Dr. Dacke and colleagues showed that all of the beetles used orientation cues to navigate their balls away from the dung source. However, the results demonstrated that the hierarchy of cues used to orient the direction which they moved the ball depended on their ecological history.

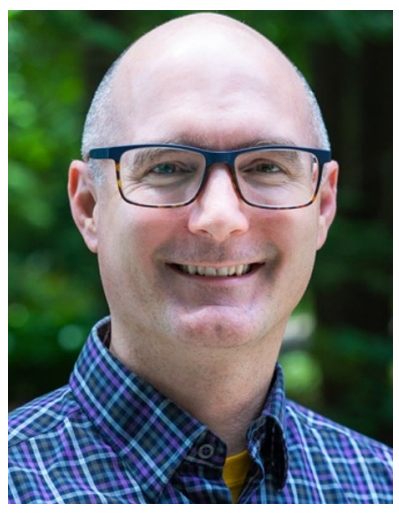

The third talk was by a researcher who would not be traditionally considered a neuroethologist. However, his results 
have interesting implications about the evolutionary role of cross-species interactions on behavior that need to be considered when trying to understand the evolutionary pressures that modify behavior. George Perry is anthropologist who gave a very intriguing talk about the role that human behavior has had in driving the evolution of other, non-domesticated species. Humans have many behaviors that lead to ecological changes, and animals must adapt to these changes or die out. Dr. Perry's review in this issue covers a number of examples of this type of adaptation in response to specific harvesting pressures, landscape modifications, and introduction of non-endemic species. Some species have been very successful in adapting to the human altered environment; others have not. One approach to understanding this suggested by Dr. Perry is genomic scans for "signatures" of selection in response to human activities in much the same way researchers have looked for similar patterns of gene changes in "tamed" or domesticated animals versus their "wild" relatives.

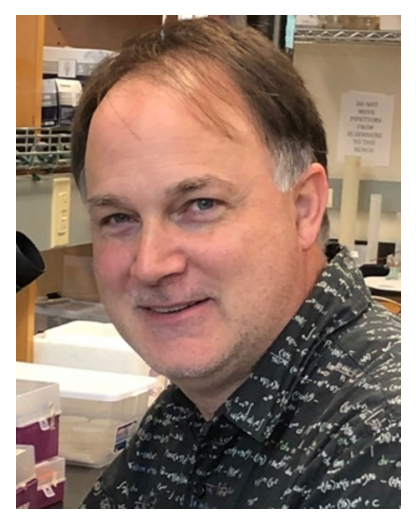

Jonathan Pierce presented data showing that the microscopic nematode, Caenorhabditis elegans can use magnetic cues to guide spatial orientation. The research report in this issue based on his talk entitled "Factors that influence magnetic orientation in C. elegans" by C Bainbridge, B Clites, C S Caldart, B Palacios, K Rollins, D Golombek, $\mathrm{J}$ Pierce, and A Vidal-Gadea is a wonderful example about the necessity to examine a behavior at multiple time-points and under multiple conditions to truly understand it. Dr. Pierce and colleagues showed that temporal, spatial, and environmental factors all influence the spatiotemporal trajectory that worms make during magnetotaxis in replicable ways. If tested early in their exposure to a specific magnetic field, worms chose one trajectory. However, after $90 \mathrm{~min}$ in a magnetic field, the preferred orientation of the worms changes. If the environment is dry (low humidity), worms show strong magnetotaxis; however, if the environment is humid, their responses to magnetic fields are less robust. The shape of the magnetic field also has strong effects on the preferred orientation of C. elegans in the field. This paper is a great example of the complex factors that alter a simple behavior, even in an organism with only 302 neurons!

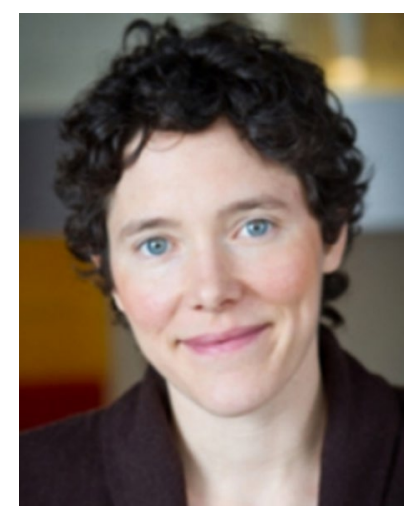

Dr. McBride's research focusses on the olfactory system of the mosquito. This review entitled "Evolution of insect olfactory circuits across short time-scales" by Zhilei Zhao and Carolyn McBride examines how insects' olfactory systems change and evolve in response to alterations in the environment including new sources of prey, and alterations in niches or competitors. This review examines potential changes at the level of olfactory receptors, olfactory neural circuits, and central olfactory processing. The authors review two separate examples of this in some depth: Drosophila sechellia flies and Heliothis moths. After reviewing these two well-studied species, they then review types of evolutionary changes found in neural circuits with examples for each type. They then move through key types of circuit evolution, cataloging examples from other insects and looking for repeated patterns of changes. Much of the literature on insect olfaction has focused on sensory neuron number and tuning, and much less attention has been paid to changes in central circuits. To date, only a few species of insects have been examined at multiple levels of circuits. The intriguing results described in this review suggest that this will be an exciting new field of research. 


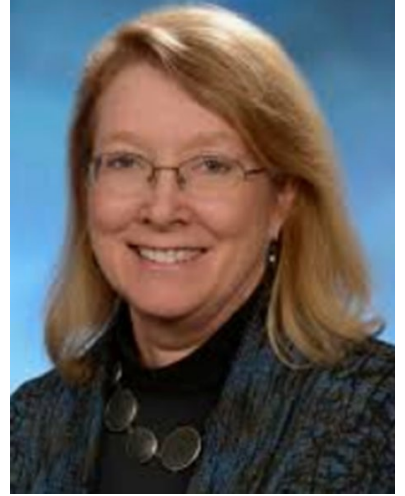

The final talk in the symposium by Margaret McCarthy took yet another new direction as she reviewed the variety of origins of sex differences in brains and behaviors, and offers a new hypothesis for the key factors that lead to sexual differentiation of mammalian brains. Her review paper in this issue entitled "A new view of sexual differentiation of mammalian brain" describes all of many factors that influence lasting sex differences in the brain. The traditional answer 20 or 30 years ago might have been hormones. However, in this fascinating review, Dr. McCarthy updates us and suggests a new hypothesis about the important roles that other factors have played in the ontogeny of sex differences in the brain. After reviewing the older literature on the role of hormones in brain sexual differentiation, Dr. McCarthy indicates that data from modern transcriptomic networks suggest that many other factors play a role in sexual differentiation of brain areas. In particular, she focusses on transcription regulatory networks and, surprisingly, immune/ inflammatory signaling and cells. Based on the data which she presents in this review, Dr. McCarthy hypothesizes a new working model of sex differentiation of the brain in which neuroinflammatory and neuroimmune factors rather than steroid hormones play critical roles in the masculinization of brain areas.

We hope the papers in this section enroll readers in the exciting field of Neuroethology and help new researchers and trainees to appreciate the intricacies and excitement of behavioral analyses and to appreciate the importance of detailed understanding of behavior before rushing to determine a mechanism. We also would like to invite readers to consider attending the next Congress of the Society for Neuroethology to experience the broad range of amazing scientific stories (you can find out more about the Society of Neuroethology and the next congress at the ISN website: https ://www.neuroethology.org/default.aspx)! The articles in this issue illustrate the complexity and sensitivity of behavior to small changes in the environment as would be expected from the ultimate read-out of the nervous system. Paraphrasing Krakauer et al. (2017) "Behavior must be seen as a meaningful endpoint and not a simple single binary unit"; for this, appreciating and understanding behavior itself are of utmost importance. Hopefully, the papers in this issue reinforce this point and stimulate researchers to value detailed behavioral analyses as critical for the forward progression of understanding neuroscience.

\section{Reference}

Krakauer JW, Ghazanfar AA, Gomez-Marin A, MacIver MA, Poeppel D (2017) Neuroscience needs behavior: Correcting a reductionist bias. Neuron 93(3):480-490

Publisher's Note Springer Nature remains neutral with regard to jurisdictional claims in published maps and institutional affiliations. 